\title{
Quantifying the effect of a mask on expiratory flows
}

\author{
Philippe Bourrianne $\odot,{ }^{1}$ Nan Xue $\odot,{ }^{1}$ Janine Nunes $\odot,{ }^{1}$ \\ Manouk Abkarian $\odot,{ }^{2}$ and Howard A. Stone ${ }^{1}{ }^{1}$ \\ ${ }^{1}$ Department of Mechanical and Aerospace Engineering, \\ Princeton University, Princeton, New Jersey 08544, USA \\ ${ }^{2}$ Centre de Biologie Structurale, CNRS UMR 5048, INSERM UMR 1054, \\ University of Montpellier, 34090 Montpellier, France
}

(Received 29 June 2021; accepted 4 October 2021; published 23 November 2021)

\begin{abstract}
Face masks are used widely to mitigate the spread of infectious diseases. While their main purpose is to filter pathogenic droplets, masks also represent a porous barrier to exhaled and inhaled air flow. In this study, we characterize the aerodynamic effect of the presence of a mask by tracking the air exhaled by a person through a mask, using both infrared imaging and particle image velocimetry performed on illuminated fog droplets surrounding a subject. We show how a mask confines the exhaled flows within tens of centimeters in front of a person breathing or speaking. In addition, we show that the tissue of common surgical face masks has a low permeability, which efficiently transforms the jetlike flows of exhalation produced during breathing or speaking into quasivertical buoyancy-driven flows. Therefore, wearing a mask offers a strong mitigation of direct transport of infectious material in addition to providing a filtering function. By comparing results on human subjects and model experiments, we propose a model to rationalize how a mask changes the air flow, and thus we provide quantitative insights that are useful for descriptions of disease transmission.
\end{abstract}

DOI: 10.1103/PhysRevFluids.6.110511

Airborne pathogens causing respiratory infectious diseases are spread by droplets carrying viruses, bacteria, or other germs emanating during breathing, speaking, singing, laughing, sneezing, or coughing of an infected person [1-9]. Airborne transmission of pathogens has been studied at least since the pioneering work of Wells and colleagues in the 1930s [10-12]. The pathogenic droplets are carried by the air flows generated by an infected person [2,13-15], before sedimenting (large drops) [2,10] or eventually remaining suspended in the air, as occurs for the smaller drops or aerosols [16-21].

Creating strategies to limit contamination requires the understanding and reduction of the extent of propagation of the respiratory air flows controlling the transport of infected droplets. During the COVID-19 pandemic, face masks were used widely to mitigate the spread of the disease, similar to the use of masks in earlier pandemics. The use of masks is recommended by health agencies, including the Centers for Disease Control (CDC) and the World Health Organization (WHO) [22], and their efficiency has been demonstrated [23-25], an approach known in hospitals [26-28] and Asian countries for many decades [29], if not centuries.

The main purpose of a mask is to filter and reduce the number of droplets emanating from a person breathing, speaking, or coughing and sneezing [30-32], and it also serves similarly to filter inhaled air [33]; in both cases, the filtration serves to reduce contamination. The relative efficiency of different masks has been compared in order to issue and improve safety guidelines [32-37]. Finally, masks also modify the air flows exhaled by a person [38-40]. 
Air flows exhaled by an unmasked person were imaged and described quantitatively during speaking [41] or during violent events such as sneezing or coughing [2,13]. In both of these cases, the air flows can propagate to distances larger than $1 \mathrm{~m}$ within one or a few seconds. These observations indicate the benefit of social distancing guidelines to avoid contamination through the direct contact by the largest droplets and by the respiratory jets carrying aerosols droplets $[42,43]$.

In addition to an effective filtration of the droplets, the use of a mask is expected to modify the dynamics of exhaled air jets, and thus reduce the short-term spread of pathogens from an infected person. Qualitative experimental visualizations have been carried out during breathing, speaking, laughing, coughing, and sneezing using a Schlieren imaging setup [38,44], or by illuminating tracer particles with a laser sheet around a human subject [45] or a model subject [46]; the flows are limited spatially while wearing a mask. However, quantifying how the air flows are modified by a mask remains to be addressed, which is the focus of our paper. Here we combine precise measurements of the expiratory flow-rates and visualization techniques able to track the exhaled air using both an infrared camera and a fog illuminated by a laser sheet.

\section{EXPERIMENTAL METHODS}

In this study, we quantify and characterize how various breathing and speaking patterns are modified by the use of a mask. By combining different imaging techniques to detect and measure the air flows around a person, we confirm the localization of the air flow around a masked individual, and, more importantly, we quantify the trajectories of the exhaled air flows. Moreover, the additional use of controlled model experiments, which are consistent with the measurements on people, allow us to rationalize the quantitative measurements. Our results demonstrate and quantify the major role played by masks to reduce drastically the speed and the subsequent propagation of exhaled air flows.

We focused our study on the most used face mask, a 3-ply surgical mask (see the Supplemental Material S1 [47]). In our experiments on human subjects, we used three complementary setups to investigate exhaled air flows: two were imaging techniques to track the air flows, and a third measured the expiratory flow-rate.

\section{A. Imaging of exhaled carbon dioxide}

First, we used a high-speed infrared camera (FLIR X6900SC) that tracks the warm exhaled $\mathrm{CO}_{2}$. The use of a filter in the absorption range of $\mathrm{CO}_{2}(4.2 \mu \mathrm{m})$ and a high-performance cooled sensor operating in the midwave $(1.5-5 \mu \mathrm{m})$ range of the infrared spectrum enables tracking the exhaled $\mathrm{CO}_{2}[48,49]$. Human subjects sat beside a dark, nonreflective curtain, which provides a uniform background at the ambient temperature $T_{0}=23 \pm 1{ }^{\circ} \mathrm{C}$. Additional curtains were used around and above the human subject to limit the influence of the background flow within the experimental space. As seen in supplementary Figs. S2-3 and movies S1-6 (or in Fig. 1 with false colors), the warm face and warm exhaled $\mathrm{CO}_{2}$ of the human subject are visible in contrast with the dark background. This technique allows us to track the exhaled $\mathrm{CO}_{2}$ as a tracer of the exhaled air flows. However, both thermal homogenization and air mixing decrease the concentration of the warm exhaled $\mathrm{CO}_{2}$ over time. Thus, the exhaled $\mathrm{CO}_{2}$ ultimately reaches the limit of sensitivity of the infrared camera at large distances and/or timescales. We validate the $\mathrm{CO}_{2}$ detection with a direct visualization technique of the exhaled air with a laser sheet, as described next.

\section{B. Imaging exhaled flows with a laser sheet}

Similar experiments were performed with a second kind of visualization using a high-speed camera (Phantom v7.3) to record the flow field induced in an artificial fog [41]. The air was seeded with small droplets from a fog machine (American DJ; fog juice by Froggys Fog) and the aerosol was made visible by a green laser sheet $(\lambda=532 \mathrm{~nm})$ created from the top of the room in a vertical plane centered on the face of the human subject wearing laser safety glasses during the experiment. Curtains were placed around the person to limit the influence of the background flow in the room. 
Moreover, we waited around 1 min after dispensing fog droplets prior to our measurements in order to homogenize the concentration of droplets in the field of view and avoid any residual air flows generated by the fog machine. As seen in Fig. 2(d) and movie S7, the background flow is $O(1) \mathrm{cm} / \mathrm{s}$.

The laser sheet technique enabled tracking the air flows exhaled from the mouth and the nose of the person, as the exhaled air is darker than the bright fog, as shown in Fig. 2. By analyzing data with particle image velocimetry (PIV) techniques, the deformation field of the fog in the plane illuminated by the laser sheet was measured to provide a two-dimensional picture of the local flow velocities. The combination of the infrared camera (3D visualization) and fog visualizations (2D quantification) offers complementary views of the exhaled air flows. Taken together, the results validate the use of $\mathrm{CO}_{2}$ as a tracer of the exhaled air flows.

\section{Measuring the flow rates}

Finally, we used a flowmeter [50] to measure the respiratory flow rate $Q(t)$ of the human subject. The flowmeter is connected with medical corrugated air tubing to a cardiopulmonary resuscitation (CPR) mask (MCR Medical), covering both the nose and mouth of the human subject without leaks. The flow rates exhaled and inhaled were measured for four different kinds of respiration, representative of a diversity of breathing patterns. Two "soft" respiration modes were investigated, one with a closed mouth ["soft (nose)"] and one with an open mouth ["soft (mouth)"]. Both represent respirations that an individual might experience while resting, in the absence of any strenuous effort or physical activity. Also, two much stronger forms of respirations were studied: A heavy breathing (denoted "heavy") consists of a series of rapid swift exhalations that approximate peak physical activity. Finally, so-called "blowing" produces a fast air jet, e.g., typical of the act of blowing out candles. Measurements of the air flows were taken on two different human subjects (one male and one female) without any major difference in the results obtained (see the supplementary Figs. S2-S3). The measurement of the corresponding respiratory flow rates reproduce the distinctive breathing patterns. The flow rates $Q(t)$ in Fig. 1(c) were measured in the absence of a mask. We qualitatively probe the influence of a mask on $Q(t)$ in supplementary Fig. S4.

\section{RESULTS}

\section{A. Masked versus unmasked individuals}

Infrared images of the exhaled $\mathrm{CO}_{2}$ and corresponding measurements of the flow rates $Q(t)$ for the four types of respiration for one human subject are presented in Fig. 1 with false colors. Similar black and white figures are provided in the Supplemental Material S2-3 for both humans participating in this study. The four cases are shown in Fig. 1 in the order of increasing magnitude of the strength of respiration: "soft" from the nose (i, blue) or with an open mouth (ii, red) to heavy breathing (iii, green) and, finally, blowing (iv, orange). For contrast, infrared imaging of a human breathing without a mask is displayed in Fig. 1(a). The face of the person saturates the infrared footage, but the warm exhaled $\mathrm{CO}_{2}$ can still be detected.

All of the static views in Fig. 1(a) demonstrate that exhaled unmasked flows lead to the formation of jets (see, e.g., [41]), which are able to reach distances of several tens of centimeters or even $1 \mathrm{~m}$ within a second for many types of exhalations, including speech. The trajectories of the jets depend on the type of respiration, as the air emanating from the nose while the mouth is closed (i, blue) is directed downwards whereas the exhaled air from the mouth (ii, red) is directed mostly in front of the person; additional visualizations are provided in the supplementary movies S1-2.

The same experiment was repeated with a 3-ply mask (see S1) as shown in the closeup views in Fig. 1(b) and in supplementary movies S3-4. The warm $\mathrm{CO}_{2}$ is now confined to a much smaller distance near the human subject. This response is evident for soft respirations (i and ii) as a weak cloud of $\mathrm{CO}_{2}$ can only be seen within about $1 \mathrm{~cm}$ around the mask. The $\mathrm{CO}_{2}$ cloud does not take the form of a respiratory jet but remains in a thin layer homogeneously spread around 

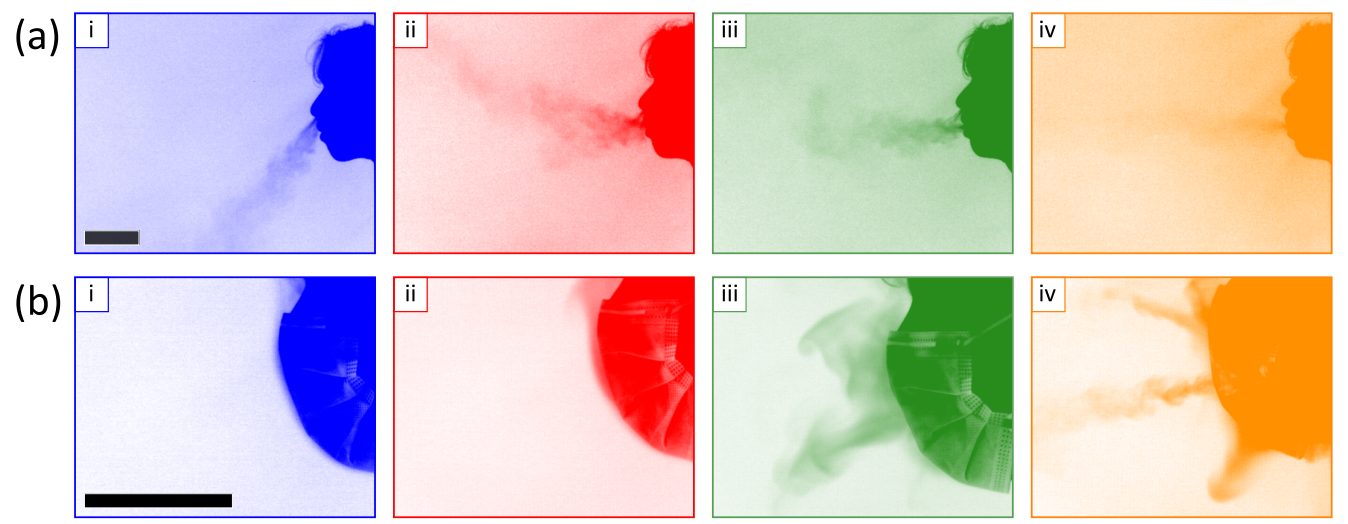

(c)
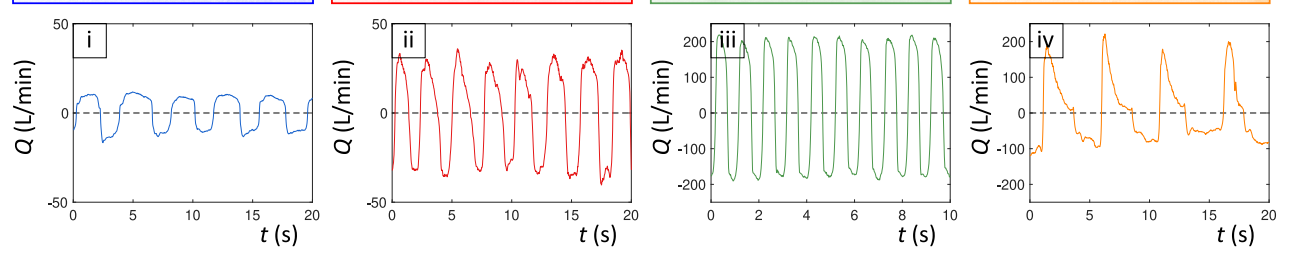

FIG. 1. Typical air flows during four distinct exhalations without and with a face mask. (a) Visualization of the exhaled $\mathrm{CO}_{2}$ without wearing a mask (false colors). From left to right: (i, blue) soft respiration from the nose, (ii, red) mild respiration with an open mouth, (iii, green) heavy respiration, and (iv, orange) blowing. The scale bar represents $10 \mathrm{~cm}$. (b) Visualization of the exhaled $\mathrm{CO}_{2}$ for the four kinds of exhalations (i)-(iv) when the subject wears a mask (false colors). While exhaled flow is contained within $1 \mathrm{~cm}$ from the mask in the case of soft respirations (i) and (ii), it reaches distances of the order of $10 \mathrm{~cm}$ for heavy respiration (iii) and (iv) as $\mathrm{CO}_{2}$ jets emanate from the mask. The scale bar represents $10 \mathrm{~cm}$. (c) Measurements of the expiratory flow-rate $Q(t)$ during those four kinds of respirations. Depending on the kind of respiration, the expiratory flow-rate $Q$ reaches a maximum at approximately $15 \mathrm{~L} / \mathrm{min}$ (i, blue data), $40 \mathrm{~L} / \mathrm{min}$ (ii, red), or $200 \mathrm{~L} / \mathrm{min}$ (iii-iv, green and orange). The horizontal dashed line delimits exhalations $(Q>0)$ and inhalations $(Q<0)$.

the mask and the face. A secondary cloud of warm exhaled $\mathrm{CO}_{2}$ is observed at the top of the mask, suggesting the presence of leaks around the nose. In contrast, more intense respirations (iii and iv) generate heterogeneous flows and the formation of several jets. While the jets reach distances around $10 \mathrm{~cm}$, which is an order of magnitude larger than the directed flows reported for masked soft respirations, they remain much more localized than their equivalent in the absence of masks [41]. The comparison between Figs. 1(a) and 1(b) highlights the physical effect of a mask to mitigate expiratory flows as reported by previous experimental studies for other kinds of situations $[38,44-46]$.

The respiratory flow rates $Q(t)$ for the four kinds of respirations are reported in Fig. 1(c). Successive respirations are performed to demonstrate the ability of the human subject to repeat similar breathing for the different experiments. The flow rates $Q(t)$ oscillate between negative values $(Q<0)$ for inhalation and positive values for exhalation. The maximum value of the flow rate scales from $15 \mathrm{~L} / \mathrm{min}$ (soft respiration from the nose), to $40 \mathrm{~L} / \mathrm{min}$ (soft respiration with an open mouth), up to $200 \mathrm{~L} / \mathrm{min}$ for both heavy breathing and blowing. The frequency of respiration also varies depending on the experiment, which leads to different volumes of exhaled air. More characterization of the measurements can be found in supplementary Fig. S5.

The dynamics of the air flows can be seen in supplementary movies S1-4 obtained with an infrared camera. The speed of the flows emanating from a mask is reduced drastically by comparison to the respiratory flows emanating from a person without a mask, in large part because unmasked exhalation is in the form of a directed jet, which is to be contrasted with the more spherical "source" 

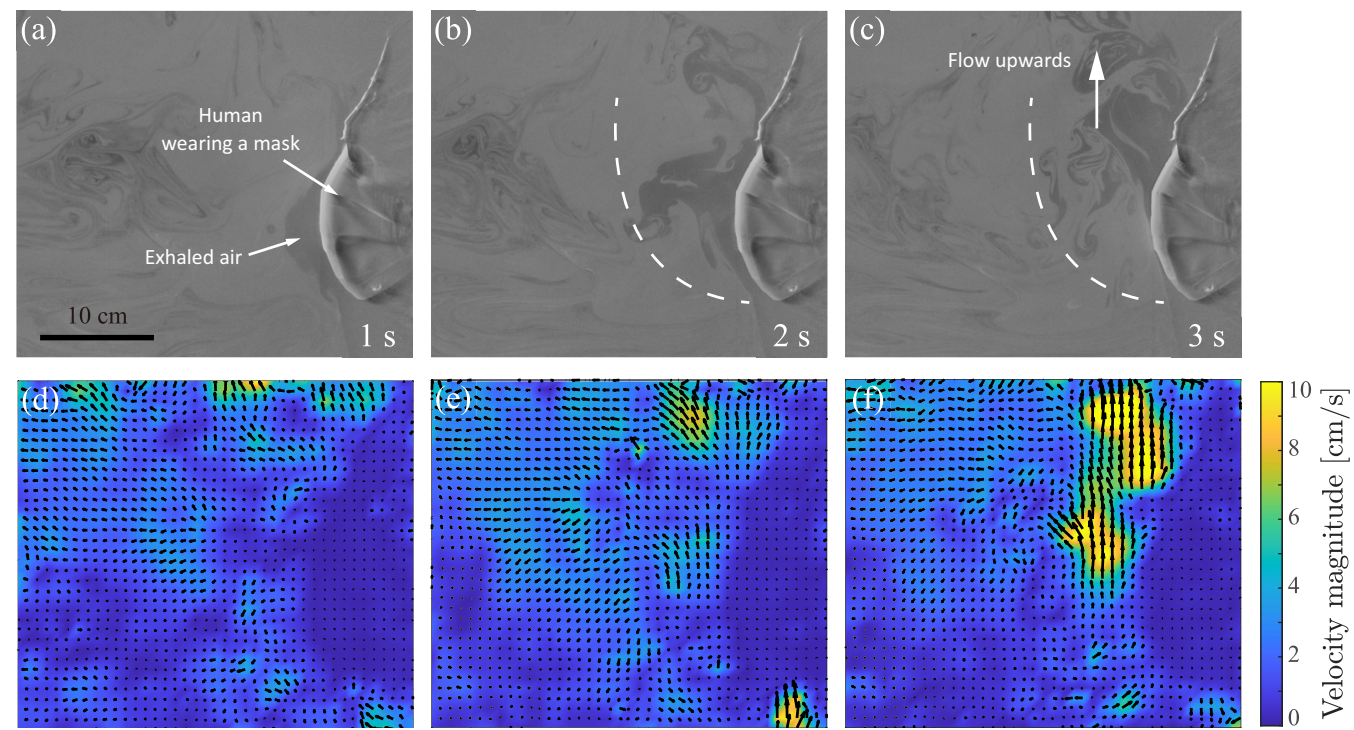

FIG. 2. Mask wearing induces localized buoyant flows during respiration. (a)-(c) Snapshots of normal breathing, 1,2, and $3 \mathrm{~s}$ after exhalation. The exhaled air is detected as a dark cloud in a bright fog illuminated by a laser sheet. The dashed line is a guide for the eyes delimiting the approximate area affected by the ascending exhaled air. (d)-(f) Measurements of the velocity field of (a)-(c). The black arrows (and the color map) show the velocity direction and the magnitude of the flow, according to the PIV analysis. See the movie and the processed PIV results in supplementary movie S7.

flow from a mask. Moreover, in the presence of a mask, the warm $\mathrm{CO}_{2}$ rises at the end of the exhalation due to buoyancy, as mentioned in the literature $[38,44]$.

To investigate these observations more carefully, we performed experiments using a laser sheet and a fog to image and characterize the flow velocities around a person. Also, we replicated the experiments with a third human subject. In this case, the exhaled air generates a dark area due to the absence of fog droplets, as shown in Fig. 2(a), $1 \mathrm{~s}$ after the beginning of the exhalation. The fog technique allows us to track the exhaled air and supports the observations made by tracking the warm $\mathrm{CO}_{2}$ shown in Fig. 1. Due to the $2 \mathrm{D}$ limitations of the area imaged with the laser sheet, it is of key importance to avoid leaks from the mask, which would lead to more complex 3D flows observed in the front views imaged by the infrared camera (see supplementary movies S5-6). Thus, in Fig. 2, the human subject wears a mask without noticeable leaks, in contrast with the images of Fig. 1(b). In the absence of leaks, the air flows during a mild respiration expand slightly more than in Fig. 1(a-i) and 1(a-ii), but the exhaled air from a masked individual remains within a few centimeters from the face, in good agreement with the $\mathrm{CO}_{2}$ measurements. After $2 \mathrm{~s}$, the air flow expands and reaches distances about $10 \mathrm{~cm}$ from the human subject [Fig. 2(b)], until it finally rises due to buoyancy [Fig. 2(c)].

The fog combined with laser sheet illumination provides a tool to measure the flow velocities of the exhaled air using the PIV technique. The experiments are processed using PIVlab [51]. The measurements are reported in Figs. 2(d)-2(f) and supplementary movie S7, and, not surprisingly, they indicate that velocities remain much slower than in the case of an unmasked individual reported in the literature [41]. The typical values of the flows, measured near the mouth, are reduced from $1 \mathrm{~m} / \mathrm{s}$ without a mask to only a few $\mathrm{cm} / \mathrm{s}$ with a mask. Moreover, the direction of the exhaled air is modified with a mask as the air rises rapidly; see, e.g., the vertical arrows in Fig. 2(f). A similar observation can be made in the case of a masked human speaking, as seen in supplementary Fig. S6 and movie S8. In all cases, the rising flows are only observed when the air is exhaled, as no clear 

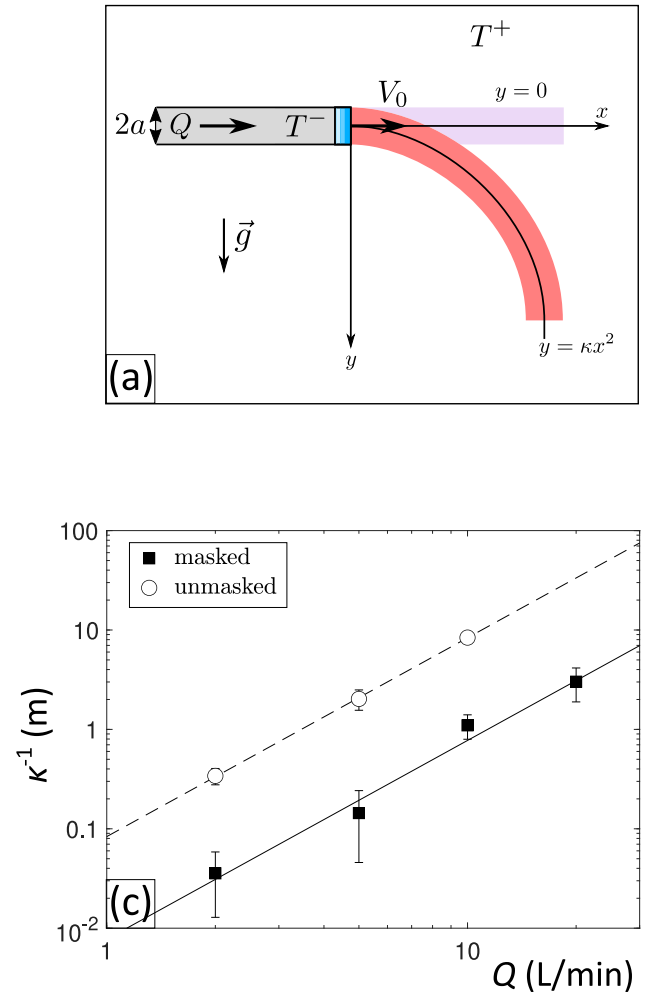
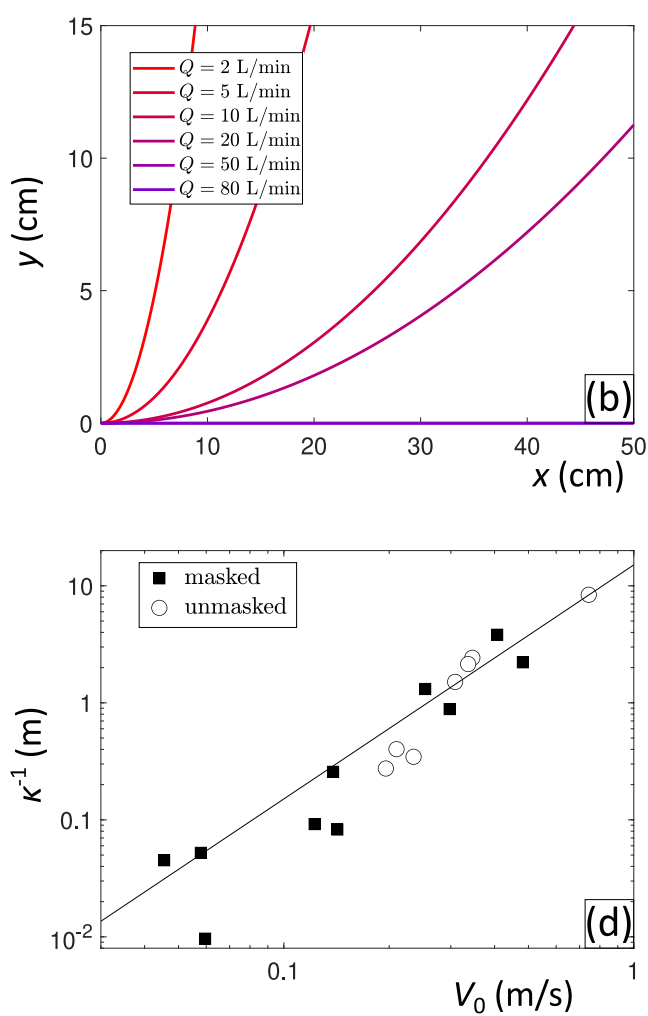

FIG. 3. Trajectory of a controlled jet of cold air. (a) Sketch of the experiment: a constant flow rate $Q$ of cold air (at the temperature $T^{-}$) is imposed for $3 \mathrm{~s}$ from a pipe of diameter $2 a$ leading to an initial velocity $V_{0}$. The pipe could be open or covered by a 3-ply mask (blue layers) sealed over the end of the tube, as seen in Fig. S7(a). Depending on the imposed flow rate $Q$, the trajectory of the jet is linear ( $y=0$, light purple) or quadratic $\left(y=\kappa x^{2}\right.$, in red). (b) Trajectories $y(x)$ of cold air jets emanating from a pipe covered by a mask as a function of the flow rate $Q(2 \leqslant Q \leqslant 80 \mathrm{~L} / \mathrm{min})$. The solid lines represent fits of the trajectories approximating the flow, leading to a quadratic shape $y=\kappa x^{2}$ for low flow rates $(Q \leqslant 20 \mathrm{~L} / \mathrm{min})$ and straight trajectories $(y=0)$ for $Q=50$ and $80 \mathrm{~L} / \mathrm{min}$. (c) Values of the radius of curvature $\kappa^{-1}$ of the jet as a function of the flow rate $Q$ with (filled squares) and without (open circles) a mask covering the pipe. The lines represent the equation $\kappa^{-1}=\alpha Q^{2}$ obtained after fitting the data without a mask (dashed line) and with a mask (solid line). The parameter $\alpha$ decreases by an order of magnitude when the pipe is covered by the mask. (d) Values of the radius of curvature $\kappa^{-1}$ of the jet as a function of the measured initial velocity of the jet $V_{0}$ with (filled squares) and without (open circles) a mask covering the pipe. All data collapse on the solid line of equation $\kappa^{-1}=\beta V_{0}{ }^{2}$.

vertical motion is noticed around the person prior to exhalations [see Fig. 2(d) and supplementary movies S7-9]. This suggests that the buoyant flows are induced by the difference of temperature between exhaled and ambient air rather than the body plume often associated with rising expiratory flows around a masked person $[38,44]$.

\section{B. Model experiments with flow from a masked orifice}

In addition to the experiments performed on human subjects, we designed a model experiment of a masked flow, as sketched in Fig. 3(a). The experiment consists of injecting a cold air jet $\left(T^{-}=\right.$ $\left.22.5^{\circ} \mathrm{C}\right)$ into a slightly warm atmosphere $\left(T^{+}=23.5^{\circ} \mathrm{C}\right)$ in the presence of an artificial fog. The jet is created by dispensing a given flow-rate $Q$ controlled with a mass flow controller (Alicat Scientific) for $3 \mathrm{~s}$ through a pipe with diameter $2 a=19 \mathrm{~mm}$. The value of the flow-rate $Q$ injected is verified 
with the flowmeter used in Fig. 1(c) [50]. The flow rate $Q$ was varied from 2 to $80 \mathrm{~L} / \mathrm{min}$. The experiment was performed with the pipe being covered or uncovered by a 3-ply surgical mask identical to the one used for the experiments with human subjects (see S1). The mask was sealed carefully over the end of the pipe to avoid any leaks, as seen in supplementary Fig. S7(a) and movie S10.

The trajectory $y(x)$ of the cold air is tracked inside the bright fog again illuminated by a laser sheet. The front of the jet is released from a pipe at the position $x=y=0$ with an initial horizontal velocity $V_{0}$. The small difference of temperature between the injected and the ambient air leads to a difference of density $\Delta \rho=\frac{P}{R_{a}}\left(\frac{1}{T^{-}}-\frac{1}{T^{+}}\right) \approx 0.004 \mathrm{~kg} / \mathrm{m}^{3}$, where $R_{a}$ is the specific gas constant for air. The density difference mimics the inverted flow situation when warm air is exhaled by a human, except that for the latter the difference of temperature is larger, leading to a density difference one order of magnitude higher $\left(\Delta \rho \approx-0.053 \mathrm{~kg} / \mathrm{m}^{3}\right.$ assuming the exhaled air is at $37^{\circ} \mathrm{C}$ and of identical composition to the ambient air).

Depending on the values of $Q$, the trajectory of the jet is expected to be horizontal $[y=0$, light purple in Fig. 3(a)] in the case of rapid flows dominated by inertia, or curved due to gravity in the limit of smaller flow rates, i.e., smaller initial velocities [red in Fig. 3(a)]. Then, the equation of trajectory of the jet can be described approximately by a parabola $y=\kappa x^{2}$ at leading order [52]. This parabolic profile arises from a balance of inertia and buoyancy by analogy with the free fall of an object, which leads to $\kappa \approx \frac{1}{2} \frac{\Delta \rho}{\rho} \frac{g}{Q^{2}}\left(\pi a^{2}\right)^{2}$. In the presence of the mask, typical trajectories are reported in Fig. 3(b). At low flow rates $(Q \leqslant 20 \mathrm{~L} / \mathrm{min})$, the trajectories can be fitted with a parabolic profile, as shown in Fig. 3(b). However, above $20 \mathrm{~L} / \mathrm{min}$, the trajectory of the jet remains horizontal over $50 \mathrm{~cm}$, as seen in Fig. 3(b), where the trajectories of jets at $Q=50$ and $80 \mathrm{~L} / \mathrm{min}$ superimpose on the $x$-axis. The curved trajectories at low flow rates allow extraction of a radius of curvature $\kappa^{-1}$.

The radius of curvature $\kappa^{-1}$ is reported in Fig. 3(c) as a function of the imposed flow rate $Q$. The data are obtained after averaging three measurements made on three distinct jets, and the error bars represent the standard deviations. The values reported in Fig. 3(c) are obtained in the case of an unmasked pipe (open circles) and a masked pipe (filled squares). The two lines represent quadratic fits of the data $\left(\kappa^{-1}=\alpha Q^{2}\right)$, which are in good agreement with the equation of the quadratic profile from our simple model. However, the data illustrate a difference between situations with and without the mask: the coefficient $\alpha$ decreases by a factor of 10 from an unmasked pipe (dashed line) to a masked pipe (solid line). This apparent mismatch denotes a clear and noticeable influence of a mask. While the section of the pipe and the flow rate remains identical, the advection of the jet emanating from a masked pipe seems to be slower than that of a jet emanating from an uncovered pipe.

When the flow rate is larger than $2 \mathrm{~L} / \mathrm{min}$, the Reynolds number $\operatorname{Re}=\frac{\rho \mathrm{Q}}{\eta \pi \mathrm{a}} \gtrsim 10^{3}$, so the exhaled jets are expected to be near a transition to turbulence [53]. The unmasked jets emanating from an orifice have significant vorticity [supplementary Fig. S7(b)]. Conversely, in the presence of a mask, the air is forced to flow through a porous matrix, which homogenizes the velocity distribution across the jet section, and delays the mixing of the jet with the ambient air [supplementary Fig. S7(a) and movie S10]. Not surprisingly, the initial velocity $V_{0}$ of the jet reaches larger maximum values in the case of an unmasked pipe. Indeed, the measurement of the initial velocity $V_{0}$ is noticeably lower when the pipe is covered by a 3-ply surgical mask [supplementary Fig. S7(c)]. The data reported in Fig. 3(c) can be represented as a function of the measured initial velocity $V_{0}$, as shown in Fig. 3(d), where the measured values of the radius of curvature $\kappa^{-1}$ collapse onto a single line of the equation $\kappa^{-1}=\beta V_{0}^{2}$, which is in good agreement with the model described earlier.

\section{Permeability of a mask}

The homogenization of the flow velocities by a mask can be understood due to the resistance offered by the porous material. We determined the permeability of the mask by measuring the upstream pressure inside a pipe covered by a mask, similar to the previous experiment, as sketched in the inset of Fig. 4. A flow-rate $Q$ is imposed using a mass flow controller, and the air is directly 


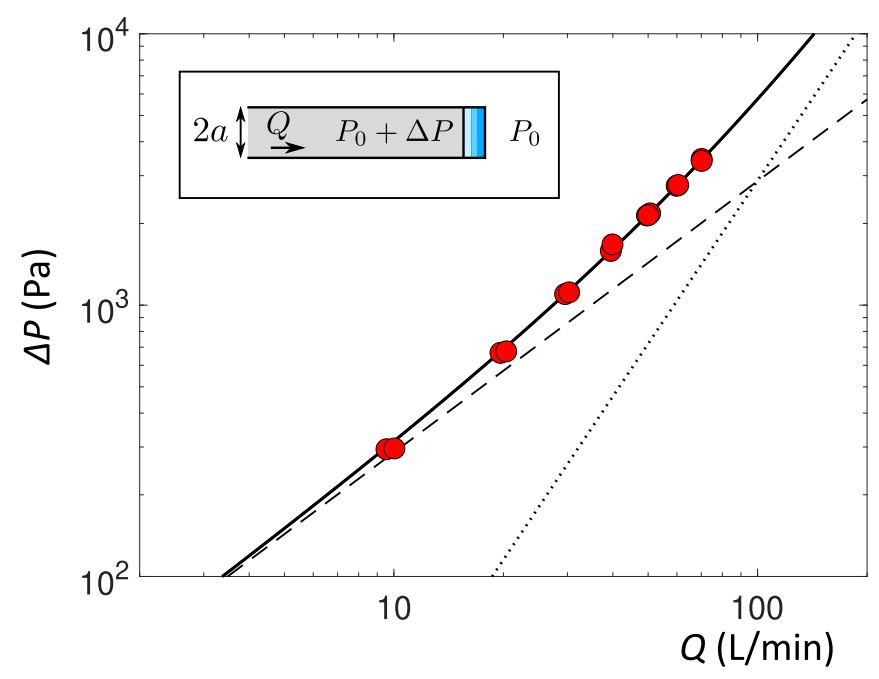

FIG. 4. Permeability of a 3-ply mask. The pressure drop $\Delta P$ across a 3-ply mask varies with the flow-rate $Q$ following the Forchheimer equation (solid line). At low flow-rates, $\Delta P$ increases linearly with $Q$ (dashed line) whereas a quadratic response (dotted line) is noticeable for increasing flow-rates. Standard deviations are within $5 \%$ of the averaged values represented by the red circles. The inset represents the sketch of the experiment. The flow-rate $Q$ is imposed through the 3-ply mask (blue layers) covering a pipe of radius $a$, and the upstream pressure $P_{0}+\Delta P$ is measured.

released into the ambient atmosphere. A pressure sensor on the flowmeter upstream reports the pressure drop $\Delta P$ across the mask; $\Delta P$ increases with the flow-rate $Q$ as shown in Fig. 4. At low flow rates, Darcy's law, which is characteristic of viscously dominated flow through a porous material, is valid as $\Delta P$ varies linearly with $Q$. At higher flow rates, $\Delta P$ varies quadratically with $Q$. The data can be fitted by $\Delta P=c_{1} Q+c_{2} Q^{2}$, which is known commonly as the Forchheimer equation [54,55], where $c_{1} \approx \frac{\eta h}{k \pi a^{2}}$ and $c_{2} \approx \frac{\rho h}{k_{i} \pi a^{2}}$, with $\rho$ and $\eta$, respectively, the density and viscosity of the air, $h$ is the mask thickness, and $k$ is the Darcy permeability, while $k_{i}$ represents a coefficient for the inertial correction. From the data, the ratio $c_{1} / c_{2}$ gives a critical value $Q^{*} \approx 100 \mathrm{~L} / \mathrm{min}$ above which the quadratic contribution dominates. Below $100 \mathrm{~L} / \mathrm{min}$, the air flow emanating from the mask is expected to be roughly laminar, as suggested by the sequence of images in Fig. S7(a). When $Q=10$ $\mathrm{L} / \mathrm{min}$, we can then deduce from $c_{1}$ a value of the permeability $k$ by considering a mask thickness $h \approx 400 \mu \mathrm{m}$ and using $a=9.5 \mathrm{~mm}$ (the radius of the pipe). From the fit in Fig. 4 , we obtain a value of the permeability $k \approx 12 \mu \mathrm{m}^{2}$.

At large flow rates, the permeability variation according to Darcy's law is not sufficient to describe the data. Indeed, above $100 \mathrm{~L} / \mathrm{min}$, the pressure drop $\Delta P(Q)$ is dominated by the quadratic component of the equation. This suggests that at high flow-rates, the mask does not laminarize the rapid turbulent flow as efficiently as at low flow-rates. Such behavior helps explain the observations made for heavy breathing and blowing in Fig. 1(b-iii) and 1(b-iv). When the expiratory flow-rate $Q$ reaches $200 \mathrm{~L} / \mathrm{min}$, the warm $\mathrm{CO}_{2}$ cloud does not form homogeneously on the entire area of the mask, but the presence of jets emanating from the mask is noticeable.

\section{Tracking the exhaled air}

Next, for the jets exhaled by the human subjects, as shown in Figs. 1 and S2-3, we track their trajectories, as shown in Fig. 5. These jets are more complicated than the model experiment as the initial orientation of the jet depends on the respiration and the position of the human subject. Figure 5(a) displays a few examples of the trajectories $y(x)$ of jets during the four types of 

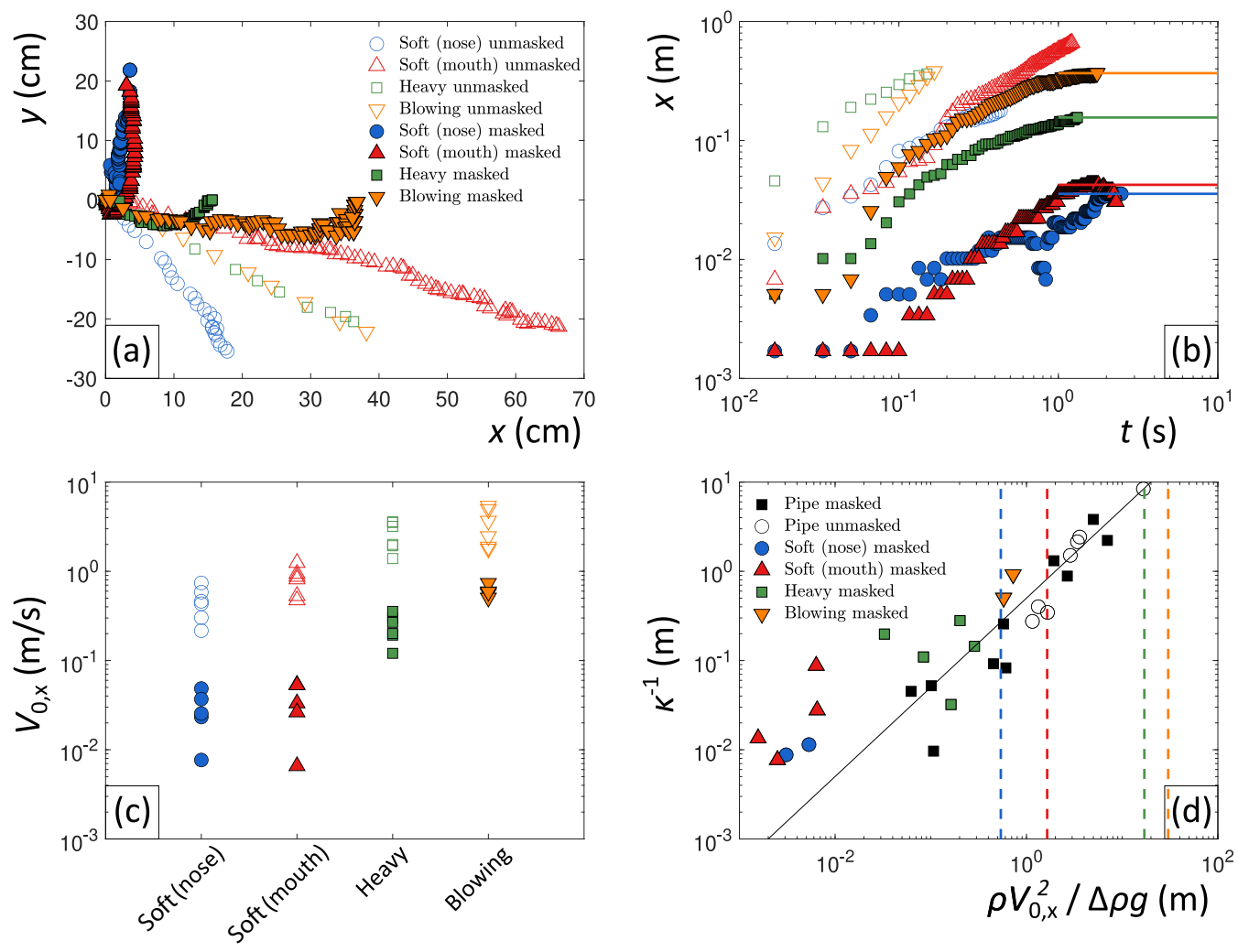

FIG. 5. Extent of the exhaled air breathed out through a mask. (a) Trajectories $y(x)$ of the front of the air jets exhaled without (open symbols) and with a mask (filled symbols). The trajectories are detected for four kinds of respirations: soft breathing from the nose (blue circles), soft breathing with an open mouth (red triangles), heavy breathing (green squares), and blowing (orange downward triangles). (b) Horizontal distance $x$ reached by the front of the exhaled air cloud as a function of time $t$ for the four kinds of respirations with and without the mask. Masked exhaled flows (filled symbols) are slower than an equivalent unmasked flow (open symbols) and reach a saturation $x_{s}$ as shown by the solid line. (c) Horizontal projection of the initial velocity with a mask (filled symbols) and without a mask (open symbols) for the four kinds of respirations. The mask leads to a substantial decrease of the initial velocity. (d) Radius of curvature $\kappa^{-1}$ for masked flows emanating from a human (colored filled symbols) as a function of the inertial-buoyant distance $\frac{\rho V_{0, x}^{2}}{\Delta \rho g}$. The data on human breathing are in relatively good agreement with the measurement with the radius of curvature $\kappa^{-1}$ on the model experiment reported in Fig. 3(d). The solid line represents a fit of the data obtained with the model experiment (black symbols) of equation $\kappa^{-1} \approx \frac{1}{2} \frac{\rho V_{0, x}^{2}}{\Delta \rho g}$, lower than the expected theoretical scaling law by a factor $\approx 4$. The dashed vertical lines represent the inertial-buoyant distance for unmasked respirations from the measured values of $V_{0, x}$ in Fig. 5(c). The expected gain in terms of radius of curvature is around two orders of magnitude.

respirations without a mask (open symbols) and with a mask (filled symbols). The human subject breathes mostly downwards while not wearing a mask, and the trajectories are inertially dominated (straight line trajectories). Conversely, for masked exhalations, the trajectories start with an initial velocity roughly aligned with the horizontal, and then they rise due to buoyancy within $30 \mathrm{~cm}$. The same pattern can be seen for the other human subject [Fig. S8(a)]. The horizontal extent $x(t)$ of the trajectories is reported in Figs. 5(b) and S8(b). The jets exhaled by an unmasked person (open symbols) propagate over the entire field of view of the infrared camera (around $60 \mathrm{~cm}$ ) within $1 \mathrm{~s}$ 
for all types of respirations, which indicates that an infected person could propagate virus-carrying droplets over $1 \mathrm{~m}$ rapidly, even at rest, without speaking, singing, sneezing, or coughing. Such measurements are in good agreement with previous measurements [13], and they highlight the possibility of an asymptomatic person spreading a virus without wearing a mask.

Conversely, the data $x(t)$ obtained while wearing a mask (filled symbols) all saturate at a distance $x_{s}$ ranging from a few centimeters for the soft respirations (blue and red horizontal lines) to a few tens of centimeters in the most violent respiration events (green and orange horizontal lines) as slow jets are still emanating from the mask. This saturation is due to the reduced flow velocity induced by the low permeability of the mask, while buoyancy redirects the exhaled air vertically.

The decrease of the initial horizontal velocity $V_{0, x}$ caused by a mask is reported in Fig. 5(c) (data obtained from two human subjects). The open symbols obtained from the jets tracked when there is no mask-wearing show much larger velocities than in the presence of a mask (filled symbols) for the four types of respirations. The initial horizontal velocity is an order of magnitude lower in the presence of the mask in the case of soft respirations. As the value of the flow-rate is constant, the size of the $\mathrm{CO}_{2}$ cloud has expanded from the typical size of the mouth $(\approx 3 \mathrm{~cm}$ in diameter) to the entire area of the mask $(\approx 10 \times 10 \mathrm{~cm})$. The velocity thus decreases by a ratio of $(10 / 3)^{2} \approx 10$, in good agreement with our measurements in Fig. 5(c). The decrease of the initial horizontal velocity is also substantial in the case of heavy breathing (green) and blowing (orange), but remains slightly less important than the factor of 10 due to the relative failure of the mask to prevent the formation of localized jets. However, the reduced velocities of the jets exhaled from intense breathing still produce a saturation in the propagation as these jets are more sensitive to buoyancy.

Recognizing the balance of inertia and buoyancy, we report in Fig. 5(d) the radius of curvature $\kappa^{-1}$ of the jet versus the inertial-buoyant distance, $\frac{\rho V_{0, x}{ }^{2}}{\Delta \rho g}$, with data from both the model experiments and on individual people. For the latter, $\kappa^{-1}$ is extracted from the trajectories $y(x)$ tracked from a human, as seen in Fig. 5(a). We find an approximate collapse of the data as a function of the inertial-buoyant distance $\frac{\rho V_{0, x}{ }^{2}}{\Delta \rho g}$ in Fig. 5(d), including from model experiments (open and filled black symbols and solid line from Fig. 3) and on the human subjects (filled colored symbols); the renormalization of the human data used $\Delta \rho=0.053 \mathrm{~kg} / \mathrm{m}^{3}$, assuming the exhaled air is released at a temperature $T^{+}=37{ }^{\circ} \mathrm{C}$ and has the same composition as the ambient air. All data approximately follow the quadratic trend described in Fig. 3. There is satisfying agreement between the model and the data, as shown by the solid line in Fig. 5(d) obtained by fitting the data obtained with the model experiment similarly to the solid line in Fig. 3(d). The radius of curvature $\kappa^{-1}$ sets the "saturation" distance $x_{s}$ reported in Fig. 5(b) and both values range from one to several tens of centimeters for masked respirations.

The results shown in Fig. 5(d) support the idea that buoyancy redirects the exhaled flows from a masked person, as noticed by previous studies $[38,44]$. However, whereas the thermal body plume of a masked person was suspected to cause the rising flows [38,44], the absence of vertical flows prior to exhalations in our PIV experiments [Fig. 2(d)] and the satisfying agreement between data on human and our model experiments without thermal body plume in Fig. 5(d) show that the difference of temperature between exhaled and ambient air directly causes the vertical redirection of the expiratory flows. In addition, no clear redirection of the flow from a person breathing was seen in the absence of a mask in the range of distances studied. However, rapid unmasked flows are also expected to rise due to buoyancy at large distances [2]. The vertical dashed lines in Fig. 5(d) represent the value of the inertial-buoyant distance expected from the value of the initial horizontal velocity $V_{0, x}$ in the absence of a mask for the four kinds of respirations [soft (nose) in blue, soft (mouth) in red, heavy breathing in green, and blowing in orange]. An increase in range between one and two orders of magnitude in terms of the saturation distance $x_{s}$ is expected and, indeed, is verified experimentally. The short-distance tracking of the jets shows that a mask inhibits the rapid propagation of exhaled air at distances longer than a few tens of centimeters. The redirection of the reduced-speed jets due to buoyancy is a systematic feature when wearing a mask. 
However, we recall from Fig. 1(b) that leaks might appear around a mask and affect this scenario. Infrared movies captured in front of a breathing person with a mask are provided in supplementary movies S5-6. These movies indicate that leaks occur on all the sides of the mask, both laterally and around the nose and chin of the human subject, as reported by complementary previous studies $[44,45]$. Tracking the leaks shows relatively slow flows, which indicates that wearing a mask remains largely aerodynamically efficient even though leaks are present; see Figs. S9-10. The leaks are, however, major failures to the efficiency of the mask as a filter of respiratory droplets [56]. In addition, the leakage flows are faster than elsewhere along a mask, leading to a larger area of possible contamination. The flow velocity of a leaking jet is larger, up to $30 \mathrm{~cm} / \mathrm{s}$, as seen in supplementary Fig. S10 and movie S9 using a fog illuminated by a laser sheet. Moreover, our results are not only valid for breathing but also for speaking (as seen in supplementary Fig. S11) in which measured horizontal distances reached in the presence of a mask are orders of magnitude lower than the rapid jets during unmasked speaking [41].

Even though our results only apply to the expiratory air flows, the tracking of the exhaled air gives information on the trajectories and positions of pathogenic droplets conveyed by the flows. However, deviations from the scenario of flow-driven transport of droplets are expected in some circumstances. First, large droplets are too heavy to be carried by the air flows, and sediment due to their own weight $[2,10]$. Then, while the motion of both aerosol droplets and the warm exhaled $\mathrm{CO}_{2}$ (tracked with the infrared camera) should follow the Taylor diffusion theory [57], deviations are expected in the turbulent regime [58,59]. For instance, weakly inertial particles are not distributed homogeneously in a turbulent flow and concentrate in strain-dominated regions [60]. Finally, the lifetime of aerosol droplets is extended by the turbulent mixing in the exhaled air flows, which maintains humid air around droplets [18]. However, a mask might reduce the contributions of these three deviations due to the efficient filtration of the largest droplets and the laminarization of the expiratory air flows by a mask.

In conclusion, our results demonstrate that face masks significantly reduce the speed of exhaled air flows, leading to a clear reduction of the spatial extent of jets potentially carrying pathogenic droplets. In addition, buoyancy then becomes dominant over the reduced inertia of the masked air flows, which contributes to an efficient redirection of the exhaled air. Finally, while our study focuses on short-time dynamics of the flows, our findings might also contribute to strategies for long-time mitigation of aerosols. As unfiltered aerosols are expected to rise due to buoyancy on shorter length scales when using a face mask, the filtration of the air above masked people, combined with well-designed ventilation, could create safer conditions for social interactions in the time of a pandemic.

\section{ACKNOWLEDGMENTS}

The authors acknowledge the Princeton Open Ventilation Monitor Collaboration for the development of the flow meter and pressure sensor used in the study. We thank Michael Roselli and Tim McDowd from FLIR for the gracious loan of the infrared camera. We thank the NSF for support via the RAPID grant CBET 2029370 and grant CBET 2116184, as well as the support of the ANR via the RA COVID grant ANR-21-CO15-0004. The study was approved by the Princeton University Institutional Review Board (protocol no. 12834).

[1] G. Johnson, L. Morawska, Z. Ristovski, M. Hargreaves, K. Mengersen, C. Chao, M. Wan, Y. Li, X. Xie, D. Katoshevski, and S. Corbett, Modality of human expired aerosol size distributions, J. Aerosol Sci. 42, 839 (2011).

[2] L. Bourouiba, E. Dehandschoewercker, and J. W. M. Bush, Violent expiratory events: on coughing and sneezing, J. Fluid Mech. 745, 537 (2014). 
[3] S. Asadi, A. S. Wexler, C. D. Cappa, S. Barreda, N. M. Bouvier, and W. D. Ristenpart, Aerosol emission and superemission during human speech increase with voice loudness, Sci. Rep. 9, 2348 (2019).

[4] P. Anfinrud, V. Stadnytskyi, C. E. Bax, and A. Bax, Visualizing speech-generated oral fluid droplets with laser light scattering, N. Engl. J. Med. 382, 2061 (2020).

[5] M. Abkarian and H. A. Stone, Stretching and break-up of saliva filaments during speech: A route for pathogen aerosolization and its potential mitigation, Phys. Rev. Fluids 5, 102301(R) (2020).

[6] T. Dbouk and D. Drikakis, On coughing and airborne droplet transmission to humans, Phys. Fluids 32, 053310 (2020).

[7] L. Morawska and G. Buonanno, The physics of particle formation and deposition during breathing, Nat. Rev. Phys. 3, 300 (2021).

[8] F. K. A. Gregson, N. A. Watson, C. M. Orton, A. E. Haddrell, L. P. McCarthy, T. J. R. Finnie, N. Gent, G. C. Donaldson, P. L. Shah, J. D. Calder, B. R. Bzdek, D. Costello, and J. P. Reid, Comparing aerosol concentrations and particle size distributions generated by singing, speaking and breathing, Aerosol Sci. Technol. 55, 681 (2021).

[9] V. Stadnytskyi, C. E. Bax, A. Bax, and P. Anfinrud, The airborne lifetime of small speech droplets and their potential importance in SARS-CoV-2 transmission, Proc. Natl. Acad. Sci. USA 117, 11875 (2020).

[10] W. F. Wells, On air-borne infection. study ii. droplets and droplet nuclei, Am. J. Epidemiol. 20, 611 (1934).

[11] W. F. Wells and M. W. Wells, Air-borne infection, J. Am. Med. Assoc. 107, 1805 (1936).

[12] W. F. Wells, Airborne Contagion and Air Hygiene: An Ecological Study of Droplet Infections (Harvard University Press, Cambridge, MA, 1955).

[13] J. W. Tang, A. D. Nicolle, C. A. Klettner, J. Pantelic, L. Wang, A. B. Suhaimi, A. Y. L. Tan, G. W. X. Ong, R. Su, C. Sekhar, D. D. W. Cheong, and K. W. Tham, Airflow dynamics of human jets: sneezing and breathing-potential sources of infectious aerosols, PloS One 8, e59970 (2013).

[14] R. Mittal, R. Ni, and J.-H. Seo, The flow physics of COVID-19, J. Fluid Mech. 894, F2 (2020).

[15] M. L. Pöhlker, O. O. Krüger, J.-D. Förster, T. Berkemeier, W. Elbert, J. Fröhlich-Nowoisky, U. Pöschl, C. Pöhlker, G. Bagheri, E. Bodenschatz, J. A. Huffman, S. Scheithauer, and E. Mikhailov, Respiratory aerosols and droplets in the transmission of infectious diseases, arXiv:2103.01188.

[16] J. L. Schulman and E. D. Kilbourne, Airborne transmission of influenza virus infection in mice, Nature 195, 1129 (1962).

[17] R. M. Jones and L. M. Brosseau, Aerosol transmission of infectious disease, J. Occup. Environ. Med. 57, 501 (2015).

[18] K. L. Chong, C. S. Ng, N. Hori, R. Yang, R. Verzicco, and D. Lohse, Extended Lifetime of Respiratory Droplets in a Turbulent Vapor Puff and its Implications on Airborne Disease Transmission, Phys. Rev. Lett. 126, 034502 (2021).

[19] A. C. Fears, W. B. Klimstra, P. Duprex, A. Hartman, S. C. Weaver, K. S. Plante, D. Mirchandani, J. A. Plante, P. V. Aguilar, D. Fernández, A. Nalca, A. Totura, D. Dyer, B. Kearney, M. Lackemeyer, J. K. Bohannon, R. Johnson, R. F. Garry, D. S. Reed, and C. J. Roy, Persistence of severe acute respiratory syndrome coronavirus 2 in aerosol suspensions, Emerg. Infect. Dis. 26, 2168 (2020).

[20] M. Richard, A. Kok, D. de Meulder, T. M. Bestebroer, M. M. Lamers, N. M. A. Okba, M. Fentener van Vlissingen, B. Rockx, B. L. Haagmans, M. P. G. Koopmans, R. A. M. Fouchier, and S. Herfst, SARS-CoV-2 is transmitted via contact and via the air between ferrets, Nat. Commun. 11, 3496 (2020).

[21] R. Zhang, Y. Li, A. L. Zhang, Y. Wang, and M. J. Molina, Identifying airborne transmission as the dominant route for the spread of COVID-19, Proc. Natl. Acad. Sci. USA 117, 14857 (2020).

[22] J. T. Brooks, J. C. Butler, and R. R. Redfield, Universal masking to prevent SARS-CoV-2 transmissionThe time is now, J. Am. Med. Assoc. 324, 635 (2020).

[23] X. Wang, E. G. Ferro, G. Zhou, D. Hashimoto, and D. L. Bhatt, Association between universal masking in a health care system and SARS-CoV-2 positivity among health care workers, J. Am. Med. Assoc. 324, 703 (2020).

[24] M. J. Hendrix, C. Walde, K. Findley, and R. Trotman, Absence of apparent transmission of SARS-CoV2 from two stylists after exposure at a hair salon with a universal face covering policy-Springfield, Missouri, May, 2020, MMWR Morb. Mortal. Wkly. Rep. 69, 930 (2020). 
[25] Y. Cheng, N. Ma, C. Witt, S. Rapp, P. S. Wild, M. O. Andreae, U. Pöschl, and H. Su, Face masks effectively limit the probability of SARS-CoV-2 transmission, Science 372, eabg6296 (2021).

[26] G. H. Weaver, The value of the face mask and other measures: In prevention of diphtheria, meningitis, pneumonia, etc., J. Am. Med. Assoc. 70, 76 (1918).

[27] D. K. Nakayama, Surgical masks during the influenza pandemic of 1918-1920, Am. Surg. 86, 557 (2020).

[28] C. Matuschek, F. Moll, H. Fangerau, J. C. Fischer, K. Zänker, M. Van Griensven, M. Schneider, D. Kindgen-Milles, W. T. Knoefel, A. Lichtenberg et al., The history and value of face masks, Eur. J. Med. Res. 25, 23 (2020).

[29] A. Burgess and M. Horii, Risk, ritual and health responsibilisation: Japan's 'safety blanket' of surgical face mask-wearing: Surgical face mask-wearing in Japan, Sociol. Health Illn. 34, 1184 (2012).

[30] T. Oberg and L. M. Brosseau, Surgical mask filter and fit performance, Am. J. Infect. Control 36, 276 (2008).

[31] L. Bandiera, G. Pavar, G. Pisetta, S. Otomo, E. Mangano, J. R. Seckl, P. Digard, E. Molinari, F. Menolascina, and I. M. Viola, Face coverings and respiratory tract droplet dispersion, R. Soc. Open Sci. 7, 201663 (2020).

[32] S. Asadi, C. D. Cappa, S. Barreda, A. S. Wexler, N. M. Bouvier, and W. D. Ristenpart, Efficacy of masks and face coverings in controlling outward aerosol particle emission from expiratory activities, Sci. Rep. 10, 15665 (2020).

[33] A. Bałazy, M. Toivola, A. Adhikari, S. K. Sivasubramani, T. Reponen, and S. A. Grinshpun, Do N95 respirators provide $95 \%$ protection level against airborne viruses, and how adequate are surgical masks?, Am. J. Infect. Control 34, 51 (2006).

[34] E. P. Fischer, M. C. Fischer, D. Grass, I. Henrion, W. S. Warren, and E. Westman, Low-cost measurement of facemask efficacy for filtering expelled droplets during speech, Sci. Adv. 6, eabd3083 (2020).

[35] A. Konda, A. Prakash, G. A. Moss, M. Schmoldt, G. D. Grant, and S. Guha, Aerosol filtration efficiency of common fabrics used in respiratory cloth masks, ACS Nano 14, 6339 (2020).

[36] G. Bagheri, B. Thiede, B. Hejazi, O. Schlenczek, and E. Bodenschatz, Face-masks save us from SARSCoV-2 transmission, arXiv:2106.00375.

[37] S. Sharma, R. Pinto, A. Saha, S. Chaudhuri, and S. Basu, On secondary atomization and blockage of surrogate cough droplets in single- and multilayer face masks, Sci. Adv. 7, eabf0452 (2021).

[38] J. W. Tang, T. J. Liebner, B. A. Craven, and G. S. Settles, A schlieren optical study of the human cough with and without wearing masks for aerosol infection control, J. R. Soc. Interface 6, S727 (2009).

[39] J. W.-T. Tang and G. Settles, Coughing and masks, N. Engl. J. Med. 361, e62 (2009).

[40] B. Maher, R. Chavez, G. C. Tomaz, T. Nguyen, and Y. Hassan, A fluid mechanics explanation of the effectiveness of common materials for respiratory masks, Int. J. Infect. Dis. 99, 505 (2020).

[41] M. Abkarian, S. Mendez, N. Xue, F. Yang, and H. A. Stone, Speech can produce jet-like transport relevant to asymptomatic spreading of virus, Proc. Natl. Acad. Sci. USA 117, 25237 (2020).

[42] M. Z. Bazant and J. W. Bush, A guideline to limit indoor airborne transmission of COVID-19, Proc. Natl. Acad. Sci. USA 118, e2018995118 (2021).

[43] F. Yang, A. A. Pahlavan, S. Mendez, M. Abkarian, and H. A. Stone, Towards improved social distancing guidelines: Space and time dependence of virus transmission from speech-driven aerosol transport between two individuals, Phys. Rev. Fluids 5, 122501(R) (2020).

[44] R. K. Bhagat, M. S. Davies Wykes, S. B. Dalziel, and P. F. Linden, Effects of ventilation on the indoor spread of COVID-19, J. Fluid Mech. 903, F1 (2020).

[45] C. J. Kähler and R. Hain, Fundamental protective mechanisms of face masks against droplet infections, J. Aerosol Sci. 148, 105617 (2020).

[46] S. Verma, M. Dhanak, and J. Frankenfield, Visualizing the effectiveness of face masks in obstructing respiratory jets, Phys. Fluids 32, 061708 (2020).

[47] See Supplemental Material at http://link.aps.org/supplemental/10.1103/PhysRevFluids.6.110511 for supplementary figures as well as text associated with each of the supplementary movie files.

[48] J. Fei, Z. Zhu, and I. Pavlidis, Imaging breathing rate in the $\mathrm{CO} 2$ absorption band, in 2005 IEEE Engineering in Medicine and Biology 27th Annual Conference (IEEE, Shanghai, China, 2005), pp. 700-705. 
[49] P. Bourrianne, P. R. Kaneelil, M. Abkarian, and H. A. Stone, Tracking the air exhaled by an opera singer, Phys. Rev. Fluids 6, 110503 (2021).

[50] Princeton Open Ventilation Monitor Collaboration, P. Bourrianne, S. Chidzik, D. J. Cohen, P. Elmer, T. Hallowell, T. J. Kilbaugh, D. Lange, A. M. Leifer, D. R. Marlow, P. D. Meyers, E. Normand, J. Nunes, M. Oh, L. Page, T. Pereira, J. Pivarski, H. Schreiner, H. A. Stone, D. W. Tank, S. Thiberge, and C. Tully, Inexpensive multi-patient respiratory monitoring system for helmet ventilation during COVID-19 pandemic, medRXiv (2020), doi: 10.1101/2020.06.29.20141283.

[51] W. Thielicke and E. Stamhuis, PIVlab-towards user-friendly, affordable and accurate digital particle image velocimetry in matlab, J. Open Res. Softw. 2, e30 (2014).

[52] E. O. Tuck, The shape of free jets of water under gravity, J. Fluid Mech. 76, 625 (1976).

[53] E. List, Turbulent jets and plumes, Annu. Rev. Fluid Mech. 14, 189 (1982).

[54] S. Irmay, On the theoretical derivation of Darcy and Forchheimer formulas, Eos, Trans. Am. Geophys. Union 39, 702 (1958).

[55] S. Whitaker, The Forchheimer equation: A theoretical development, Transp. Porous Med. 25, 27 (1996).

[56] C. D. Cappa, S. Asadi, S. Barreda, A. S. Wexler, N. M. Bouvier, and W. D. Ristenpart, Expiratory aerosol particle escape from surgical masks due to imperfect sealing, Sci. Rep. 11, 12110 (2021).

[57] G. I. Taylor, Diffusion by continuous movements, Proc. London Math. Soc. s2-20, 196 (1922).

[58] E. Villermaux and C. Innocenti, On the geometry of turbulent mixing, J. Fluid Mech. 393, 123 (1999).

[59] F. Poydenot, I. Abdourahamane, E. Caplain, S. Der, J. Haiech, A. Jallon, I. Khoutami, A. Loucif, E. Marinov, and B. Andreotti, Risk assessment for long and short range airborne transmission of SARSCoV-2, indoors and outdoors, using carbon dioxide measurements, arXiv:2106.09489.

[60] M. Gibert, H. Xu, and E. Bodenschatz, Where do small, weakly inertial particles go in a turbulent flow?, J. Fluid Mech. 698, 160 (2012). 